\title{
CONTRAPUNTO FILOSÓFICO EDUCACIONAL EN CHILE: HUMANISMO LAICO VERSUS HUMANISMO CRISTIANO
}

\author{
Jaime Caiceo Escudero*
}

\begin{abstract}
RESUMEN
Este artículo persigue poner en contrapunto dos filosofías educacionales de dos personajes de la segunda mitad del siglo XX que representan el pensamiento laico y el pensamiento católico en Chile; ellos son Roberto Munizaga y Ernesto Livacic, quienes recibieron en 1979 y 1993, respectivamente, el Premio Nacional de Educación. A pesar de las diferencias ideológicas, ambos personajes mantienen una postura antagónica respecto de las creencias religiosas, pero en cuanto a sus respectivos análisis sobre el papel que representa el proceso de educación en la vida del hombre muestran notables coincidencias. Los aspectos fundamentales de la filosofía de la educación son abordados desde sus respectivos enfoques.
\end{abstract}

Palabras claves: Filosofía de la Educación. Pensamiento Educativo. Historia de la Educación. Fundamentos de la Educación. Futuro Educacional.

\begin{abstract}
This paper's aim is to put in counterpoint to two educational philosophies of two different characters from the second half of the XX century; these philosophies represent the secular and Catholic thought in Chile. They are: Roberto Munizaga and Ernesto Livacic who received the National Prize of Education in 1979 and 1993, respectively. In spite of the ideological differences, both of them take a tough contrary stand related to religious beliefs, however when they make their own analysis of the role that the educational process take place in human beings' lives, they share outstanding concordances. The main aspects of Philosophy of education are treated from their respective approaches.
\end{abstract}

\footnotetext{
* Licenciado en Filosofía y Doctor en Ciencias de la Educación (Pontificia Universidad Católica Argentina, 1996); académico de los Postgrados en la Universidad de Santiago de Chile y en la Universidad Nacional de Cuyo, Argentina. Miembro de la Sociedad Chilena de Filosofía y Miembro Directivo de la Sociedad Chilena de Historia de la Educación. E-mail: jcaiceo@hotmail.com
} 
Keywords: Philosophy of Education. Educational Thought. History of Education. Educational Foundation. Educational Future.

\section{Introducción}

Durante la Colonia no hubo contrapeso a la fuerte y decisiva influencia que las congregaciones religiosas tuvieron en la educación chilena. En efecto, franciscanos, dominicos, mercedarios, agustinos y jesuitas trajeron la educación al país con un objetivo claramente evangelizador en una primera etapa $\mathrm{y}$, posteriormente, formativo. Las diferentes congregaciones estaban influidas por la filosofía escolástica en sus diferentes versiones: la tomista (asumida por los dominicos, los mercedarios y, en parte, por los agustinos), la escotista, propia de los franciscanos y la suarecista seguida por los jesuitas (CAICEO, 1995a).

Con el advenimiento de la Independencia en los albores del siglo XIX y de la República, posteriormente, comenzó el naciente estado a tomar importantes decisiones en el plano educacional, fundando el Instituto Nacional en 1813, la Universidad de Chile y la Primera Escuela de Preceptores, ambas instituciones creadas en 1842 y un sinnúmero de escuelas primarias, especialmente a partir del gobierno de Bernardo O'Hggins (18181823). Estas instituciones educacionales fueron rápidamente consolidándose en el país a partir del naciente laicismo que comenzaba a imponerse, fruto de los planteamientos de la ilustración, el enciclopedismo y el racionalismo que habían iluminado el espíritu libertario que condujo a la Independencia; el positivismo tuvo durante la segunda mitad del siglo XIX un fuerte influjo en el país a través de los Hermanos Lagarrigue. Así fue como desde 1860 se estableció en Chile el denominado estado docente, el cual estaba avalado por un fuerte laicismo que se fue imponiendo en el ámbito educacional. Los sectores católicos no se quedaron atrás y levantando la bandera de la libertad de enseñanza, llegaron a fundar la Universidad Católica de Chile en 1888 para detener intelectualmente el fuerte influjo laicista. Se produjo un fuerte enfrentamiento a nivel político entre la Iglesia Católica y el Estado Laico, encabezado en ese momento por representantes de esa postura (Krebs, (1981) y, a nivel educacional se agudizó más la pugna entre estado docente y libertad de enseñanza. 
Si durante el siglo XIX Chile miraba en el plano educativo hacia Europa, a partir del siglo pasado esa mirada se concentró en Norteamérica. Por lo mismo no es extraña la presencia de la filosofía educacional y la pedagogía de John Dewey en Chile en gran parte del siglo XX. Su pensamiento inspiró el debate que culminó con la promulgación de la ley de instrucción primaria obligatoria en 1920 y las Reformas de 1927 y 1945 (CAICEO, 1993; 1995b). Sin embargo, el planteamiento del gran pedagogo norteamericano que llegó de la mano de los laicos al país, especialmente a través de Darío Salas y su hija Irma fue también estudiado y profundizado por los católicos, a partir de la tesis doctoral del P. Hurtado publicada en 1935 en Lovaina (HURTADO, 1994). En esta ocasión, la corriente laicista estaba liderada por Irma Salas y la católica por San Alberto Hurtado, pero no necesariamente en enfrentamiento sino que más bien en confluencia, pues ello permitió que la Reforma de 1965 cambiara la línea de pensamiento laico que había dominado la educación chilena, desde el estado, por una de inspiración humanista cristiana.

El objeto de este artículo es poner en contrapunto dos filosofías educacionales de dos personajes de la segunda mitad del siglo XX que representan el pensamiento laico y el pensamiento católico; ellos son Roberto Munizaga y Ernesto Livacic, quienes recibieron en 1979 y 1993, respectivamente el Premio Nacional de Educación. Siendo ambos intelectuales, el segundo es también hombre de acción educativa.

\section{El Humanismo Laico de Munizaga}

El pensamiento de Roberto Munizaga abarca muchas temáticas, pero a partir de sus dos ejes centrales, la filosofía y la educación. Sin embargo, para un análisis de toda su obra, ésta se abordará en cinco áreas: Historia de la Educación e importantes Educadores; Fundamentos de la Educación; Filosofía de la Educación; Filosofía y algunos Filósofos; Universidad. Todo lo anterior se encuentra en una gran cantidad de publicaciones en un lapso de tiempo cercano a los 70 años ${ }^{1}$.

\footnotetext{
1 Si se considera la publicación de su primer poema en 1924 por Nascimento y su último libro editado en 1995 por la Editorial Universitaria han transcurrido 71 años.
} 


\section{1 Área de Historia de la Educación e Importantes Educadores}

Más que hacer Historia de la Educación, el autor que nos preocupa hizo Historia del Pensamiento Educacional Chileno desde la segunda mitad del siglo XIX hasta avanzado el siglo XX. En efecto, su preocupación principal estuvo en buscar las raíces más próximas de la educación primaria y de la educación superior en Chile; sobre la educación secundaria elaborará una filosofía educacional.

En el primer caso, pensó que Domingo Faustino Sarmiento, el educador argentino, Rector de la Primera Escuela de Preceptores, en 1842, había trazado su línea de acción, la cual se perdió con la partida del trasandino. Munizaga lo admiró mucho y no escatimó elogios para él, catalogándolo como "uno de los protagonistas más certeros de la cultura en América” (MUNIZAGA, 1937).

Al mismo tiempo, lamentó que no se hubiera continuado la línea del educador argentino y se produjera un corte entre la partida de Sarmiento y el inicio de la Reforma de 1893. Al respecto, señaló:

Bien distinto sería el estado de la instrucción pública en Chile si la tradición del gran educacionista argentino no se hubiera perdido...Si Sarmiento no hubiera sido olvidado, su obra nos hubiera hecho ir más rápidamente de Pestalozzi a Wundt, del empirismo a la ciencia, de los maestros natos a los que técnicamente se forman, del diletantismo al profesionalismo en la enseñanza (MUNIZAGA, 1937, p. 29 y ss.).

Munizaga lamentó lo que había sucedido en la Escuela Normal, situación que conocía de cerca por su experiencia como docente; en forma enfática, concluyó que no se había seguido con la línea educacional trazada por Sarmiento:

Hemos pasado de una a otra dirección extranjerizante en la organización de nuestros estudios y en la formación de nuestros maestros. Lo cual explica, sin duda, excelentes enriquecimientos en la técnica menuda de la labor en la sala de clases, pero, al mismo tiempo, es responsable de esa sensación general de incoherencia, ese aire sonámbulo, 
automático, de curioso despego respecto a las necesidades del país con que nos impresiona, a veces, el funcionamiento de nuestro sistema total de enseñanza (MUNIZAGA, 1937, p. 29 y ss.).

En síntesis, a su juicio, la educación primaria es la base del sistema educativo por donde todos deben comenzar para llegar a los niveles siguientes del sistema escolar; está en contra de una escuela primaria para pobres y un colegio para las clases acomodadas, distanciando y no uniendo a las diferentes clases sociales; la escuela puede y debe jugar un rol protagónico para favorecer la unidad nacional inter-clases sociales.

En el segundo caso, Valentín Letelier es su modelo y quien, según su parecer, ha hecho un mayor aporte para ubicar a la educación universitaria en el sitial que le corresponde. Sin embargo, al igual como sucedió con Sarmiento, piensa que se produjo una discontinuidad en el devenir educacional chileno, de ahí, la crisis existente en la Universidad. Valoraba profundamente el ideario de Letelier cuando acotaba:

cuya obra filosófica, pedagógica y política, de tan definidos contornos, sirve como ninguna para ilustrar el aberrante fenómeno de la discontinuidad de nuestra cultura latinoamericana, y, especialmente chilena (MUNIZAGA, 1969, p. 68).

Cuando ejerció la Presidencia del Instituto de Chile, Munizaga creó la Colección de Educadores Chilenos de Ayer y de Hoy, publicando él mismo textos sobre varios importantes educadores nacionales, tales como Darío Salas, Oscar Vera, los Hermanos Amunátegui, Claudio Matte, Valentín Letelier, Alejandro Venegas, José Abelardo Núñez, Amanda Labarca y Gabriela Mistral; todos, salvo la última, son exponentes del laicismo en Chile, tanto en su expresión positivista - seguidores de Comte - como pragmática - continuadores de Dewey.

\section{2 Área de Fundamentos de la Educación}

Como buen filósofo y científico de la educación, Munizaga inició su incursión en la educación, buscando definir el concepto e indicando 
sus objetivos en términos originales; sin embargo, junto a su experiencia personal estaba toda la influencia recibida de filósofos y educadores, tanto nacionales como extranjeros, indicados en el punto anterior y en los tres que siguen. De esta forma, precisó que la educación

se identifica con el esfuerzo de cualquier organismo social para perseverar en su esencia mediante la renovación de su propia vida. Podría decirse, analógicamente, que la educación equivale a una verdadera 'función sexual' de la comunidad. En efecto, ella es la que permite que su tipo de existencia y su fisonomía cultural - un sistema de principios, una imagen del hombre y una tabla de valores -, se reproduzcan y prolonguen en la materia plástica de las generaciones nuevas (MUNIZAGA, 1965, p. 28).

A partir de la definición anterior, enfatizó que la escuela es el vaso comunicante entre el hombre y la sociedad. Estos factores claves de la educación pueden ser analizados, a su juicio, desde tres perspectivas: la biológica, la psicológica y la sociológica; a partir de ellas, surgen los que denomina tres conceptos científicos de la educación, correspondiendo en realidad a los objetivos de la educación: Socialización de las nuevas generaciones, adaptación al ambiente y desenvolvimiento de la personalidad por medio de la cultura (MUNIZAGA, 1965, p. 215).

En todo lo anterior se da el proceso de transmisión y reconstrucción de la experiencia, como algo propio de la educación, puesto que

queremos destacar que ellos son orientaciones fundamentales del espíritu, que de inmediato colorean y definen cualquiera situación pedagógica, de la escuela primaria a la universidad (MUNIZAGA, 1965, p. 37).

E1 Profesor Munizaga abordó una serie de problemas educacionales a partir de los fundamentos anteriores; entre otros, cabe mencionar la educación refleja, la educación valórica, el hombre culto como resultado del proceso educativo ${ }^{2}$, la dualidad entre teoría y práctica en el quehacer

2 La educación, a su juicio, debe transformar a los jóvenes al terminar su proceso formativo 
educacional, la función social de la educación y el rol activo del Estado, como centro aglutinador de un grupo humano, teniendo como instrumento a la educación. Munizaga es uno de los primeros educadores que subraya la educación refleja, describiéndola "como la acción permanente del medio sobre el individuo, en cuanto moldea todos los aspectos de nuestra personalidad, sin que tengamos de ello una clara conciencia" (MUNIZAGA, 1965, p. 37). Este tipo de educación la compara con la educación formal, concluyendo, al igual que Dewey, que toda renovación educacional debería buscar un equilibrio entre ambas, entre formalismo y realismo, entre la cultura por la escuela y cultura por la vida (CELIS; SÁNCHEZ, 1992, p. 217).

En cuanto a la educación valórica, inspirado en Max Scheler y Nicolai Hartmann, importantes filósofos contemporáneos dedicados a la Axiología, indicó que

el sentido del hombre en el mundo se haya dado por su misión de realizar valores, la cual hace que exista una dinámica correlación entre la estructura metafísica del universo y la textura de la vida personal, concluyendo que la educación es un proceso de realización de valores (MUNIZAGA, 1965, p. 140).

A juicio de Roberto Munizaga, la dualidad teoría práctica se puede superar si se reconstruye "la idea de las humanidades", lo cual quiere decir que:

equivale a superar la concepción aristocrática que durante largo tiempo las identificó unilateralmente con el estudio de las letras, ampliado más tarde con el de las ciencias, y que hoy se niega a admitir que también las técnicas u ocupaciones útiles constituyen una oportunidad de enriquecimiento intelectual y moral (MUNIZAGA, 1958, p. 81).

Respecto a la función social de la educación, indica que ésta no sólo ayuda a socializar a las nuevas generaciones transmitiendo pasivamente

en seres cultos, es decir, preparados intelectualmente para saber vivir, tal como se expone al fin del punto siguiente. 
un esquema pre-existente sino que activamente ayudará a transformarlo. Señalaba que la escuela es:

una reconstrucción de la experiencia o de la cultura, en cuanto ella se verifica sobre la materia plástica e informe de las nuevas generaciones"; por lo tanto, la escuela no se limitará a reproducir "servilmente lo que existe según la presión de las viejas estructuras que se han consolidado y detenido... su función propia, frente al medio social, consistirá entonces, más que en confirmarlo, en complementarlo y, sobre todo, rectificarlo activamente (MUNIZAGA, 1958, p. 36 y ss.).

En relación al rol del Estado no estaba de acuerdo con la libertad de enseñanza, tal como lo planteaba la Iglesia Católica, pues ella proponía a la familia como el primer agente del proceso educativo $\mathrm{y}$, a juicio de Munizaga, ello, a lo único que conduce es a la desintegración de la sociedad; textualmente, indicaba:

conduce, en último análisis, a suscribir la doctrina de que las fuerzas de desintegración han de tener mayor importancia que las de cohesión social (MUNIZAGA, 1953, p. 29).

Nuestro autor, por lo tanto, fue un gran defensor del estado docente porque ello, a su juicio, era lo único que favorecía la integración social; en los que se oponían había

mucho de espíritu anárquico en la habitual desconfianza de los individuos hacia el Estado, sobre todo, en el prurito de vivir criticando su función docente (MUNIZAGA, 1965, p. 59).

Munizaga desconfiaba del rol educativo de la familia porque en nuestro medio no había una clara concepción de la vida como en Atenas o Inglaterra. Textualmente, señalaba:

Pero en nuestras informes repúblicas latinoamericanas, tierras de aluvión, en que familias, regiones y clases, son unidades centrífugas y dispersas, ello equivale a formular, sin declararlo, un voto por la desintegración de la vida nacional (MUNIZAGA, 1965, p. 59-60). 


\section{3 Área de Filosofía de la Educación}

Abordó la educación secundaria a partir de un análisis filosófico en consideración a que cuando fue escrito el texto atingente (1944, aunque se publicó en 1947) este nivel de la educación en Chile aparecía con una fisonomía borrosa, sin que existiera claridad hacia dónde se quería ir, se daban señales contradictorias (MUNIZAGA, 1994, p. 15-16). Frente a ello, efectúa un largo análisis histórico, tanto nacional como internacional, para concluir que es necesario asumir un nuevo concepto de educación secundaria, el cual debe perseguir la formación más plena del hombre, preparándolo para la vida (humana, cívica y económica), pero también para el ingreso a la Universidad; ello debe hacerse sin entrar en contradicción con la democracia. Más aún, cuando regresó de su viaje a Estados Unidos reforzó su idea - tomada del espíritu de Dewey en Democracia y Educación: Hay que universalizar la educación secundaria para afianzar la democracia. Humanismo y democracia se complementan y no se contraponen; también hay que superar el antagonismo entre intelectualismo y economicismo que, a propósito del fin de este nivel de enseñanza ha existido por cerca de un siglo en Chile (MUNIZAGA, 1994, p. 114-124). Por lo mismo y en síntesis, señaló:

Propiciamos la creación de una nueva escuela secundaria chilena, con una personalidad propia frente a los demás géneros de enseñanza, segura de sí misma - especialmente, frente a la técnico-profesional - que, abierta sin distinción a la masa de los adolescentes, conduzca, distribuya y seleccione para construir una república dirigida por la virtud y la inteligencia - por una nueva inteligencia, formada dentro de unas humanidades nuevas (MUNIZAGA, 1994, p. 127).

Las ideas fuerzas que le atribuyó al nuevo tipo de hombre que era necesario que formara la educación secundaria, son tres: cabeza clara con espíritu lúcido (para ello son necesarias las asignaturas propias de las humanidades, especialmente el idioma patrio; las ciencias y las que ayudan a comprender las ocupaciones útiles); un corazón bien puesto que ayude a formar personas cultas con sensibilidad afinada y modales 
(para lograr esto el Liceo debe transformarse en un ambiente adecuado); y una voluntad firme con jóvenes inteligentes, pero de carácter (para ello, todas las asignaturas deben estar al servicio de los jóvenes para que ellos con su experiencia de vida en el ambiente del Liceo tengan un auténtico crecimiento moral) (MUNIZAGA, 1994, p. 127-139).

En el análisis anterior queda de manifiesto que Munizaga estuvo fuertemente influido por Dewey y su Escuela Nueva y, así como Darío Salas en El Problema Nacional aplicó estos planteamientos a la educación primaria, Munizaga hizo lo propio en relación a la educación secundaria. Sin embargo, su fuerte énfasis en el desarrollo de la inteligencia denota el positivismo de Valentín Letelier, que tanto repercutirá en nuestro autor.

Hizo también una incursión en la Filosofía de la Educación de Froebel para resaltar que con la creación del kindergarten se dio fuerza al primer peldaño de un sistema educativo que debe atender al hombre en su más pleno humanismo. Por ello, afirmará:

Más allá de las perturbaciones transitorias - tan aptas para ocasionar el desconcierto-, adivinamos la posibilidad de un efectivo reinado del hombre. Este reinado no será posible sino sobre la base de crear condiciones sociales y pedagógicas que permitan el completo desenvolvimiento de los individuos, el rendimiento total de la planta humana. Afirmarlo es renovar hoy lo mejor del espíritu de Fröebel (MUNIZAGA, 1986, p. 53).

\section{4 Área de Filosofía y Algunos Filósofos}

Fiel a la corriente laicista, Munizaga incursionará en la filosofía del inspirador del positivismo en Chile y que perduró en gran parte de la segunda mitad del siglo XIX, el filósofo francés Augusto Comte; lo mismo sucederá con las nuevas tendencias imperantes en la primera mitad del siglo XX: sus ojos y su mente mirarán hacia Norteamérica y, específicamente, se detendrá en John Dewey, catalogado por él mismo como el filósofo de América. De igual forma, destacará a sus representantes chilenos: Valentín Letelier, en el primer caso, y Darío Salas en el segundo. Sin embargo, más que centrarse en la filosofía propiamente tal de los autores antes 
mencionados, hará un análisis educacional aplicado a la realidad chilena, a partir de los principios filosóficos de aquéllos.

Munizaga ha reconocido que sus categorías de análisis para la educación y su historia las asimiló a partir de los planteamientos de Letelier. Sobre la importancia de este autor en Chile indicó:

Conviene reiterar, que el positivismo no fue completamente, entre nosotros, una ideología de imitación o un simple ejercicio de evasión. Muy al contrario, orientó la política y la enseñanza, esas dos grandes formas de la vida humana. Y, sobre todo, contribuyó a educar a las mentes criollas, extraviadas en lo teológico o lo metafísico, a rectificar su disposición viciosa, propicia a evadirse en un mundo de principios y abstracciones, ayudó a afinar la mirada para ver y sentir la realidad del mundo hispanoamericano: instalarse efectivamente en medio de sus cosas, que eso fue y pudo continuar habiendo sido entre nosotros, lo más cordial del espíritu positivo (MUNIZAGA, 1969, p. 72).

Comte y Letelier en directo se encuentran en la cita anterior. Munizaga, a su vez, defendió a Letelier de los ataques que había recibido por ser considerado un intelectualista superado por el nuevo planteamiento educacional que ligaba a este quehacer con la economía; él mismo se defendió indicando que si en vez de preocuparse del desarrollo del intelecto de los jóvenes debería dejarse a éstos al arbitrio de sus instintos. Concluyó, al respecto:

la posición humanista de Letelier - y no la economista - es la única que puede honorablemente sostener un maestro que no quiera ponerse en contradicción con la ética de su magisterio (MUNIZAGA, 1943, p. 22).

El problema anterior se había suscitado por la controversia entre la formación general y la formación específica de los educandos. Munizaga, siguiendo a Letelier, defendía que la educación amplia del hombre debía efectuarse antes que la formación específica en lo técnico; la educación primaria cumplía esa labor formativa general, pero la educación secundaria también, como corolario de aquélla; ésta última estaba llamada a formar una verdadera conciencia humana, ayudando a humanizar la técnica. 
A su vez, el laureado que nos preocupa, indicó claramente la influencia recibida por Dewey. En efecto, en el Prefacio de la edición efectuada en 1946 de una de sus obras, afirmó:

en una actitud amplia - abierta a todas las corrientes del espíritu - sin adherir a ninguna tendencia pedagógica aislada, aún cuando sería torpe dejar de reconocer la importancia que atribuimos a J. Dewey, el más grande de los filósofos de nuestro tiempo (MUNIZAGA, 1965, p. 28).

Las ideas de progreso vigentes en el país, no sólo por la teoría positivista, sino que también por la mayor riqueza de la sociedad chilena y la esperanza de ser un país desarrollado para el primer Centenario de la Independencia, favoreció la aceptación de las ideas del pensador norteamericano; sus creencias, traducidas en 1908 por Darío Salas - el Credo Pedagógico de Dewey - favorecían la intención de vincular, como lo hacía efectivamente Dewey, la ciencia y la tecnología en el esquema educacional. Favorecían la aceptación de este educador sus planteamientos de respeto a la ley, el suscitar el trabajo, la iniciativa individual y la prescindencia religiosa; todo acorde con el esquema político liberal y de sociedad laica imperante en quienes tomaban las grandes decisiones en el país a comienzos del siglo pasado. El Estado podía impulsar este modelo educacional eficiente.

Munizaga validará su proximidad con Dewey cuando tajantemente afirmó:

El hombre culto es el que se forma simultáneamente a través de la vida y de la escuela, del trato con las cosas y con los libros, extrayendo de todas partes las substancias que le permiten enriquecer su experiencia y desenvolver una personalidad genuina: la cultura no es un saber inerte, sino como se expresa la verdadera vida humana (MUNIZAGA, 1965, p. 50).

La incursión que el autor hizo en Kierkegaard está fuera de su lógica laicista. Sin embargo, ello es coherente con su preocupación de explicarse el fenómeno religioso y habla bien de su altura intelectual. El 
abrazó los principios de la masonería, pero fue bautizado y gran conocedor de la biblia, incluso la leía permanentemente en los últimos años de vida, según testimonio de Pino (2002, p. 82); fue contrario a la Iglesia Católica e intentó cooperar con la reducción del poder de ésta, especialmente en el ámbito educacional, pero deseaba racionalmente explicarse la raíz del existencialismo - imperante en su época - y sobre todo, el efecto que la fe religiosa produce en la vida y en el pensamiento. Su racionalismo, ¿se deberá a la fuerte influencia intelectual del positivismo de Letelier?

En su momento histórico, Munizaga pensó que en Chile había sólo dos auténticos filósofos, Jorge Millas y Luis Oyarzún; los demás, son profesores de filosofía, acotó (PINO, 2002, p. 64).

\section{5 Área acerca de la Universidad}

Llevaba tan sólo cinco años como académico en la Universidad cuando Roberto Munizaga, junto al Prof. Yolando Pino Saavedra, efectúa una compilación de visiones sobre la Universidad en diferentes países, tales como Alemania, Francia, Estados Unidos y Argentina, agregando un ensayo sobre Chile, escrito por él mismo. Correspondía a su primera publicación - salvo los poemas de juventud publicados en la década anterior - indicando con ello su preocupación por este nivel de enseñanza, en el cual permanecerá por más de 50 años. El título del texto es muy sugerente porque La Crisis de la Universidad ya nos conduce a una situación problemática. En efecto, con la Reforma de 1927 y la Contrarreforma de 1928, la Universidad había perdido terreno - al no poseer en adelante la tuición de la educación secundaria - y no mejoraba su calidad, pues se crearon los Institutos Universitarios para desarrollar la ciencia de cada saber y las Escuelas para aplicar el saber a las profesiones, sin embargo nada de ello ha perdurado.

Siguiendo a Max Scheler postulará que en la Universidad se deben "conservar y mantener los más altos bienes de la cultura y del saber" en donde debe darse una buena enseñanza, complementada con "investigación y formación espiritual multilateral” (CELIS EN SÁNCHEZ, 1992, p. 239). Sin embargo, cuando presentó el concepto de esta institución recurriría a José Ortega y Gassett. Afirmó, al respecto: 
Se entenderá por Universidad strictu sensu, la institución en que se enseña al hombre medio a ser un hombre culto y un buen profesional (ORTEGA Y GASSET en MUNIZAGA et al., 1933, p. 131).

Siguiendo al autor español, postulará una Facultad de Cultura como núcleo central de una Universidad, teniendo alrededor de aquélla las cinco áreas de la vida académica, a saber, Física (Imagen física del mundo), Biología (Los temas fundamentales de la vida orgánica), Historia (El proceso histórico de la vida humana), Sociología (La estructura y funcionamiento de la vida social) y Filosofía (El plano del universo).

A la visión anterior, le agregará las perspectivas de Valentín Letelier. A partir de todo ello, inició su crítica al señalar que los estudios universitarios tenían una orientación técnico profesional, olvidándose de los elementos propios de la cultura y de la ciencia; al no existir investigación, no había desarrollo científico. Asumió, a su vez, una postura que indicaba que la política no debía mezclarse con el rol y el quehacer universitarios; sin embargo, la Universidad debía responder a los requerimientos sociales del momento; por lo mismo, textualmente señaló:

Para ello tiene la Universidad que intervenir en la actualidad como tal Universidad, tratando los grandes temas del día desde su punto de vista propio - cultural, profesional y científico (ORTEGA Y GASSET en MUNIZAGA et al., 1933, p. 126).

Las acciones que debía realizar la Universidad para cumplir sus objetivos, siguiendo los ideales reformistas de Córdoba, eran:

Asimilación del patrimonio acumulado en las distintas esferas del conocimiento por los pueblos que marchan a la cabeza de la investigación científica... (lo cual supone) una profesionalización de la enseñanza universitaria, que ella no signifique una labor periférica en torno a otras actividades distintas que constituyen el eje de la vida del maestro (ORTEGA Y GASSET en MUNIZAGA et al., 1933, p. 131).

La reelaboración en la forma más personal posible de cuanto se ha pensado y escrito, trabajo regular de interpretación y comentario, 
confección de textos didácticos superiores ${ }^{3}$, aproximación a las fuentes mismas del conocimiento (ORTEGA Y GASSET en MUNIZAGA et al., 1933, p. 131).

La investigación científica propiamente tal.

En relación a la autonomía universitaria pensaba que ella debía existir en la medida que no sirviera para mantener el conservadurismo y evitar cualquier intromisión en el cumplimiento de las acciones señaladas en el párrafo anterior. Autonomía sí, pero para desarrollar la ciencia libremente. Por ello, al reflexionar sobre la libertad de cátedra y libertad de investigación, se preguntará:

¿Existe algún antagonismo entre la libertad de cátedra, divisa propia de la enseñanza superior, y la organización de la docencia universitaria, según el modo tradicional de las escuelas profesionales?

¿Existe algún antagonismo entre la libertad de la ciencia y la organización del trabajo científico, bajo la forma de centros o institutos de investigación, como lo viene realizando, por ejemplo, la Universidad de Chile? (MUNIZAGA, 1964, p. 15).

En el mismo documento de la cita anterior subraya que la ciencia es un convidado reciente a las universidades latinoamericanas y cuando llegaron los aires reformistas a Chile en 1968, se expresó en términos irónicos y con espíritu crítico, pues allí no estaban presentes sus ideales de Universidad; el título del texto que hizo circular por la Universidad en ese tiempo es decidor: Crisis y Desplome de la Universidad.

\section{El Humanismo Cristiano de Livacic}

\subsection{Antecedentes generales}

Para entender el pensamiento educativo y la acción pedagógica de Ernesto Livacic hay que tener presente cuatro influencias muy directas que

\footnotetext{
3 Para cumplir este objetivo que se trazó él mismo, Munizaga publicó especialmente sus dos obras claves: Principios de Educación y Filosofía de la Educación Secundaria.
} 
marcaron su rumbo, a saber, su familia, su colegio, el Hogar de Estudiantes y el Instituto Pedagógico.

Los padres de don Ernesto eran personas de poca educación sistemática - el padre tuvo sólo dos años de educación formal y la madre cuatro - sin embargo poseían un gran tesón que les permitió desenvolverse adecuadamente en su medio; el padre logró dominar tres lenguas (croata, español e inglés -esta última la aprendió en su trabajo, pues los dueños del frigorífico en que trabajaba eran ingleses). Asistían regularmente con sus hijos a la misa dominical que era realizada por sacerdotes salesianos; por ello, resultó natural la selección del colegio para Ernesto cuando llegó el momento. La Región de Magallanes fue evangelizada por los salesianos; por ello, en la actual Diócesis de Punta Arenas, el Obispo es un sacerdote salesiano y la mayoría de las parroquias, escuelas y colegios están a cargo de la Congregación de Don Bosco. Manuel Canales (1997, p. 133) resume muy bien los aspectos familiares y educativos del futuro laureado en los siguientes términos:

Su padre y su madre debieron realizar, en conjunto, enormes esfuerzos y sacrificios en favor de su resguardo tanto hogareño como escolar, quienes premunidos de un profundo espíritu de generosidad y abnegación a toda prueba, supieron superar, a la postre, las condiciones moderadamente modestas en que se desenvolvían. No obstante, dichas limitantes no fueron en sí impedimentos reales para que, en ese hogar, reinara una genuina alegría familiar, fortalecida por la presencia testimonial de sus padres, reconocidos por todos sus vecinos como honestos, austeros y trabajadores (CANALES, 1997, p. 133).

$\mathrm{Su}$ colegio fue una verdadera escuela de formación humana, cristiana y social. Los sacerdotes salesianos no sólo administraban el establecimiento educacional sino que también hacían las clases y programaban las diferentes actividades complementarias; usando lenguaje de la reforma educacional chilena de la última década del siglo pasado, los salesianos formaban una verdadera comunidad educativa con sus alumnos y apoderados, profundizando los objetivos verticales de cada asignatura con rigor académico y los objetivos transversales formativos que atravesaban 
todo el currículo, tanto programático como extraprogramático; de esta manera los alumnos eran formados en la confianza, la responsabilidad, la libertad, el compromiso y la fe religiosa. Esta formación se complementaba con la acción social hacia el exterior del colegio, mostrándoles la pobreza y las carencias del prójimo; se les hablaba de la necesidad de la justicia y la paz en un mundo en plena Segunda Guerra Mundial. En este ambiente, el joven Ernesto llegó a destacarse por sus méritos humanos, académicos y cristianos. Cuando ejerció el cargo de Subsecretario de Educación viajó especialmente en esa calidad a Punta Arenas y visitó a su antiguo colegio que para él fue efectivamente la continuación de su hogar.

Su paso por el Hogar de Estudiantes fue muy relevante. En efecto, allí no sólo pudo practicar diariamente su fe con la eucaristía sino que, además, en la lectura, la oración, el compañerismo y la asesoría permanente de grandes sacerdotes que marcaron las generaciones del 30 y del 40 en el siglo pasado en Chile con su rol como Asesores de la Acción Católica ${ }^{4}$. El propio Ernesto Livacic recuerda en los siguientes términos la labor de Mons. Larson en el querido Hogar:

Desde el primer momento no se dio tregua para formar cristiana e intelectualmente a los jóvenes que vivían en el Hogar y estudiaban en el Pedagógico, donde reinaban los laicos. Los resultados fueron óptimos: creó círculos de estudio sobre las verdades religiosas, realizó retiros espirituales y conferencias pedagógicas y fomentó la diaria convivencia, especialmente en el comedor: alrededor de la mesa conversaban y discutían los problemas que habían oído en el Instituto (CAICEO EN CELIS et al., 1988, p. 219).

La Universidad de Chile puso el sello final en nuestro laureado. El Instituto Pedagógico de la época era un centro de formación de profesores de excelencia y la vida estudiantil se constituía en el mejor lugar de formación cívica. Allí, el joven de la ciudad austral, se convirtió en un alumno destacado en lo académico y en un importante dirigente estudiantil

4 Cumplieron ese rol Mons. Oscar Larson, fundador de la Acción Católica en 1931 y San Alberto Hurtado entre 1941 y 1944. 
que aportaba nuevas ideas para unir las diferentes carreras en un solo local y hacer las renovaciones pedagógicas necesarias. Su participación en la Unión de Estudiantes Católicos de Pedagogía -UECP- le ayudó a preocuparse más de lo gremial y a entender que es conveniente vivir en una sociedad integradora y pluralista; no hay que olvidar que la línea inspiradora de la educación chilena en esa ápoca era laicista y librepensadora y el joven Ernesto luchaba por la implantación del humanismo cristiano; quizás el haber vivido en aquella realidad educacional le permitió sortear con éxito su aporte a la Reforma Educacional de 1965: Haber ayudado a incluir el humanismo cristiano como línea inspiradora del sistema educacional público chileno.

Los escritos de Ernesto Livacic son en sus inicios de orden literario, y su producción más significativa desde la perspectiva educacional se encuentra desde 1958 en adelante, en donde fue sistematizando su pensamiento de educador cristiano que implícita y explícitamente ponía en práctica, tanto en su labor educativa en el aula como en su actividad gestionadora de la educación pública.

Sus escritos revelan una preocupación preferente por la educación en sus diferentes aspectos desde una perspectiva cristiana y por la persona de los educandos. En efecto, sus obras sobre Literatura están pensadas para que sirvan de instrumento o medio educativo para los alumnos, ya sea a nivel universitario (la Antología, especialmente) o secundario (sus textos para este sector educacional); sus reflexiones educativas que se encuentran en libros o revistas apuntan a diferentes problemáticas o temas educativos, como la educación particular, la educación nacional, la educación por el arte, el planeamiento educacional, educación y participación, educación y desarrollo, los valores y fines de la educación, el proyecto educativo, la misión de la universidad y la educación cristiana en cuanto implica innovación y cambio en la sociedad actual. Sus escritos, a su vez, son una expresión, como causa o como efecto de su praxis educativa, ya sea en el aula universitaria o en los cargos de gestión educacional que ha ejercido. 


\subsection{Su concepción educacional}

\subsubsection{Génesis y fundamentos}

El pensamiento educacional de Ernesto Livacic, tanto en su génesis como en sus fundamentos, está atravesado integralmente por los principios del cristianismo que él fue asimilando desde su hogar y, posteriormente, en su colegio, en la universidad y en su vida adulta. En relación a la influencia determinante de su hogar ella ya ha sido expuesta precedentemente; algo similar ha sucedido con la importancia de su colegio en su concepción educacional; baste agregar que la impronta salesiana está marcada por el carisma de Don Bosco, el cual cuando ejercía su rol educador entregaba confianza a sus alumnos y siempre tenía palabras dulces, fáciles y atrayentes para relacionarse con ellos; situación que asimiló muy bien el discípulo salesiano y practicó en su labor pedagógica. Su paso por la Universidad y el Hogar de Estudiantes también ya ha sido señalado, pero es necesario agregar la importancia del pensamiento educacional de San Alberto Hurtado en don Ernesto; él mismo recuerda:

Me sorprendió al leer en la década de los 40, cuando estaba por iniciar mis estudios de Pedagogía, una afirmación del P. Hurtado al comienzo de su Libro Puntos de Educación, en el sentido de que ésta -la educación- constituiría la gran preocupación del siglo XX, así como para el anterior había sido la cuestión social y como para cada centuria había habido la propia en su momento. Parecía una posición en extremo idealista por parte de quien se había doctorado muy joven en aquella disciplina en Europa y se dedicaba a la formación de la juventud (Caiceo en Hurtado, 1994). El ritmo de los tiempos conlleva más bien, según creíamos, una clara tendencia en procura de la meta del desarrollo, entendida bajo variados prismas: el auge económico, la participación social, la modernización cientifíco- tecnológica... Cada una de estas facetas, claro está, proyectaba sus exigencias sobre la educación... Hoy, cincuenta años después de aquella lectura, nadie discute acaso que la educación consiste en la formación integral de la persona humana, y que si ella carece de todo pedestal realista no es posible tener el sueño de construir el desarrollo, porque éste sólo 
será integral en la medida que tenga como base la plenitud humana (LIVACIC, 1995, p. 14).

Como puede apreciarse, está muy viva en él la impronta de San Alberto Hurtado, como asimismo, su recuerdo revela que leyó y meditó bastante en su juventud al sacerdote jesuita que, además, fue su director espiritual.

En su vida adulta, a su vez, ingresó, junto con su esposa, al movimiento de Schoenstatt (1954); allí, ambos encontraron la paz espiritual y la motivación para una vida cristiana más plena; el objetivo del P. José Kentenich, su fundador, es que cada persona pueda buscar la formación de un nuevo hombre para una nueva sociedad. Desde la década del 50 participaron activamente en el grupo de matrimonios; don Ernesto tuvo la ocasión en dos oportunidades de reunirse con el fundador del movimiento en Alemania $(1966 ; 1967)$ y conversar sobre temas educacionales (Uriburu, 1972). En los últimos años, hasta la muerte de la Sra. Bety, permanecieron en el movimiento en calidad de Peregrinos.

También en su vida adulta fue muy influido en su pensamiento educativo porel Magisteriodela Iglesia Católica, tantoporlos planteamientos emanados del Concilio Vaticano II, como de las Conferencias Generales del Episcopado Latinoamericano realizadas en Río de Janeiro, Medellín, Puebla y Santo Domingo. Sintetiza, a su juicio, dicha influencia el $N^{o}$ 1.025 del Documento de Puebla:

La educación humaniza y personaliza al hombre cuando logra que éste desarrolle plenamente su pensamiento y su libertad, haciéndolos fructificar en hábitos de comprensión y de comunión con la totalidad del orden real por los cuales el mismo hombre humaniza su mundo, produce cultura, transforma la sociedad y construye la historia ( $\mathrm{Cfr}$. GS 55).

Lo anterior no sólo impregnó su pensamiento y su acción educacionales sino que le ha permitido hacer aportes para el desarrollo $\mathrm{y}$ difusión del pensamiento cristiano en educación, teniendo un rol de importante asesor en esta materia de la Conferencia Episcopal de Chile; su 
opinión era muy valorada por los señores Obispos (Bravo, 2000). Pero en su acción pública practicó muy bien su cristianismo cuando en calidad de Subsecretario de Educación apoyó la extensión de la Reforma de 1965 a los niños discapacitados, pues los más necesitados requieren de mayor apoyo; el pensamiento educativo de Ernesto Livacic está también atravesado por la Doctrina Social de la Iglesia; por lo mismo, ha expresado:

A todo educador acucia en forma quemante la 'opción por los pobres' en su propio medio educativo. Los pobres son todos los que padecen necesidades, desde las económicas hasta las existenciales. Y ellas se dan en todos los sectores sociales y culturales. Cada educador ha de percibir y atender las necesidades de 'sus' pobres, de los pobres entre sus educandos (LIVACIC en SALMAN et al., 1993, p. 195).

Su compromiso con la educación cristiana lo ha llevado a ser un gran defensor de la libertad de enseñanza en Chile, tema muy sensible para la educación particular, puesto que hasta hace poco más de tres décadas, la mayoría de los establecimientos educacionales particulares, tanto pagados como subvencionados, pertenecían a instituciones ligadas a la Iglesia Católica.

\subsubsection{La educación como proceso centrado en la persona}

Consecuentemente con las fuentes de su pensamiento educativo, el centro de su preocupación será la formación de la persona del educando con las implicancias que ello conlleva; no hay que olvidar que el concepto de persona equivale a la visión cristiana del ser humano. El concepto clave que atraviesa el cristianismo es el amor, pues Dios creó todo por amor, pero especialmente al hombre; por ello, para don Ernesto, la presencia del amor en la educación es fundamental; literalmente expresa:

Amar al hombre desde la educación supone insoslayablemente buscar al hombre que Dios quiere (LIVACIC en MASSONE, 1998, p. 7).

En otro pasaje señala: 
Estos dos rasgos lucen con particular fuerza en el hombre: es su creatura predilecta, la que más amó, la única a la cual dotó de la capacidad de proseguir su propia obra creadora. Dios sembró en el hombre potencialidades que éste debe desarrollar, poner en acto. En ese poner en acto las potencialidades humanas, consiste, en último término, educarse: etimológicamente, extraer de sí, 'e-ducere' (LIVACIC, 1982, p. 145).

En síntesis, una educación centrada en la persona debe formar integralmente al hombre, tal como Dios lo creó (LIVACIC, 1982), a todos los hombres sin distinción alguna (LIVACIC, 1982) y a cada hombre en su singularidad (LIVACIC, 1982); para lograr esto último, el educador debe ser creativo, facilitando la electividad de las lecturas, la libertad de temas de creación e investigación, la formación de equipos flexibles entre compañeros, la inscripción voluntaria en talleres, la rotación en la integración de comités, etc. (LIVACIC en SALMAN, 1993, p. 186).

Estrechamente ligado a esta concepción de educación se encuentra el que ella debe darse como un proceso interpersonal y valórico. Interpersonal porque es la persona del educador la que ayuda a desarrollarse a la persona del alumno en un proceso de comunicación constante; nuestro laureado escribe al respecto:

La Educación es un proceso de comunicación. Estrictamente hablando, nadie es educado por otro -ya que es sujeto de su propia formación-, pero tampoco nadie se educa solo. Ante la UNESCO, en 1980, S.S. Juan Pablo II recordó que la Educación es, en su proceso y en su proyección, esencialmente interpersonal. La educación es un diálogo. Y un diálogo tiene muchas formas posibles, y las modalidades de comunicación entre los seres humanos son también plurales, sin duda, pero no puede dejar de llamarnos a reflexión el que, si hemos recibido la palabra como don exclusivamente nuestro, a ella ha de competer un papel de particular importancia, en ese hacerse en y con otros (LIVACIC, 1996b, p. 17).

Valórico porque el educador con su ejemplo va mostrando los valores que el educando, poniendo en ejercicio su inteligencia y voluntad, 
va eligiendo e internalizando. Para ello, el educador debe poner en práctica diversas modalidades de interacción personal, tales como la disponibilidad, el gesto, la atención, la efectuosa preocupación (LIVACIC en SALMAN, 1993, p. 180).

Una educación centrada en la persona, a su vez, no puede confundir los medios con los fines. Es muy importante la elección de los medios pedagógicos para educar, pero sin olvidar que ellos están al servicio del fin fundamental de la educación: alcanzar el desarrollo pleno de la persona humana. Al respecto, Ernesto Livacic acota:

Nos parece imprescindible concordar, como punto de partida, en que, sin perjuicio de que procuremos una amplia visión del tema educacional, hemos de reconocer que su centro, su sujeto y su objeto es el propio ser que se educa. Todo lo demás, - sistemas y técnicas, objetivos y materiales de apoyo -, está al servicio de ese delicado trabajo de construcción de una personalidad y de una vida (LIVACIC en SALMAN, 1993, p. 178).

\subsubsection{Educación y participación}

Una auténtica educación es aquella que respeta el pluralismo educacional, fruto de la participación de todos los involucrados en su gestación. Este tema le preocupó bastante al Premio Nacional durante el gobierno de Salvador Allende en Chile, momento en el cual se deseaba implementar la Escuela Nacional Unificada - ENU -.Livacic, al respecto, reflexionó:

En Chile no se concibe ni tiene posibilidad alguna de prosperar una reforma educacional que no sea explícitamente consultada a instancias comunitarias (LIVACIC et al., 1973, p. 52).

Esta reflexión también es válida para el proceso iniciado en la década pasada. Los profesores se quejan constantemente a través de sus organizaciones de la poca participación que han tenido en esta última Reforma Educacional; sería conveniente que las instancias pertinentes del gobierno tuvieran en cuenta las observaciones que en su momento, ante 
otra situación histórica, pero tan válidas hoy como ayer, hiciera nuestro Premio Nacional.

Prosigue, nuestro autor, en su defensa de la participación social en la constitución de nuestro sistema escolar, con un fuerte énfasis en la educación particular y en la libertad de enseñanza, cuestionada en el gobierno de Salvador Allende:

En lo fáctico, es discutible, ciertamente, que lo precedentemente expuesto signifique que la orientación general del sistema educativo haya surgido realmente de la participación social. Sin embargo, nadie podría discutir que ha existido efectivamente, que se ha reconocido invariablemente, la diversidad de opciones de aplicación de los grandes principios comúnmente aceptados: el desarrollo de la educación particular, la vigencia de la autonomía universitaria, la multiplicidad de experiencias educativas legal y administrativamente válidas para todos los efectos, son, entre otras que podrían citarse, algunas de las más claras muestras de la libertad de enseñanza en Chile (LIVACIC et al., 1973, p. 47).

Enfatiza la importancia de la educación en la constitución de una sociedad democrática y participativa, recalcando la libertad humana como un elemento intransable en una sociedad pluralista:

En el paso de una sociedad de dominación a una sociedad de participación, corresponde a la educación un papel indudablemente señero. Ella es el elemento que más puede contribuir a liberar al hombre y a la sociedad, dentro del esquema social actual que, aunque imperfecto, es el único posible claustro materno de la nueva realidad a que aspiramos. En ese sentido, ya la incorporación al sistema educativo es, en sí, un primer paso en el camino de construcción de los cimientos de una participación social (LIVACIC et al., 1973, p. 61).

Para entender en su real dimensión histórica lo anteriormente señalado, hay que recordar los momentos críticos vividos en la sociedad chilena entre las décadas del 70 y del 80, en que primero se temió una dominación marxista para luego entrar a un régimen militar de facto, en que se conculcaron las libertades públicas. 


\subsubsection{Rol del docente}

El profesor, de acuerdo a la visión cristiana de la educación que posee Ernesto Livacic, debe intentar lograr que el educando se personalice con todo lo que el concepto implica y que ha sido precedentemente señalado. Una buena síntesis del pensamiento del autor al respecto es la siguiente:

¿Cuál es, entonces, el papel del educador?

- No el de ideologizar, presentando ideas preconcebidas de la realidad, porque ello es atentar contra la dignidad humana y contra la autonomía del saber, sino el de mostrar objetivamente su disciplina, alimentando en sus discípulos la pasión por la verdad y la relación de lo que se aprende con el hombre y su destino trascendente (LIVACIC et al., 1979, p. 72).

Por ello, más adelante señala:

Que el educador sea testigo de los valores que propone; en otras palabras, que en él se dé y se perciba un 'ser y hacer valóricamente fundados y coherentes entre sí', como ha sido definida la sabiduría; y que los educandos vivencien esos valores, hoy, en la cotidianeidad de su proceso formativo y, capten así su aplicabilidad y fecundidad, convenciéndose de ellos y abrazándolos (LIVACIC, 1996a, p. 112).

Además, ve al educador como un activo protagonista de la historia, como un agente de la misma, en su labor con los alumnos:

el educador se consagra al encauce de la historia estimulando en sus protagonistas la adhesión a las ideas y valores inmutables en una vivencia actual y experiencial que fecunde el porvenir... El educador no es, claro está, el conductor visible de la historia de su pueblo. Más que a las figuras estelares que ocupan los grandes titulares o los primeros planos de las pantallas, se asemeja a la abeja obrera en el colmenar de la intrahistoria... Cada educador es padre de una abigarrada proyección intrahistórica nutriente de la macrohistoria, 
aunque ésta muchas veces, la fagocite o la succione. Cada educador, reconocido o no en su obra, es un agente del devenir (LIVACIC, 1995, p. 12-13).

En su quehacer pedagógico, don Ernesto practicó en plenitud el rol de educador y maestro que él impulsaba en sus escritos. Transmitía un saber impresionante, con rigor académico, para luego con diversas lecturas que cada alumno podía elegir, permitir su desarrollo personal; era en extremo exigente, pero muy justo en la calificación (PALAZUELOS, 2000).

\subsubsection{Formación del docente y misión de la Universidad}

Cinco son los pilares sobre los cuales, a juicio de Livacic (1995, 1996a, 2000) debe cimentarse la formación de un educador:

- La Filosofía de la Educación para tener claro ¿qué es educar? y ¿para qué educar?Una sólida antropología filosófica es impresindible.

- La Psicología del Desarrollo y del Aprendizaje para entender al educando en su situación real de crecimiento y en su capacidad de aprendizaje.

- Antropología Cultural y Social para entender el medio en el cual vive el educando.

- Una sólida formación en la especialidad del educador.

- Metodologías generales y específicas.

Lo anterior, sin embargo, no lo ve claro en el actual proceso de formación de profesores por parte de las universidades; dice. al respecto:

La Filosofía de la Educación, la Psicopedagogía, la Antropología Cultural y Social, suelen brillar por su ausencia, o en el mejor de los casos ser parientes pobres, dentro de los currículos de formación inicial de profesores, con lo cual el producto de ésta suelen ser unos técnicos que toman el rábano por las hojas. Es explicable que haya ocurrido así durante los lustros en que nos rigieron quienes consideraban peligroso 
que pensáramos, pero es difícilmente explicable la lentitud con que se ha estado volviendo al buen camino después de que la hora de aquellos ya pasó (LIVACIC, 2000, p. 121).

\subsubsection{Desafíos hacia el siglo XXI}

Al recorrer los principales hitos de la historia educacional del siglo $\mathrm{XX}$, Ernesto Livacic describe los avances que en cobertura ha habido en el país, pero reconoce que los desafíos para el futuro se encuentran en la modernización de la educación y en el mejoramiento cualitativo de la misma; en ello es concordante con los planteamientos educacionales del estado de Chile a contar de la década del 90: Mejorar la calidad y la equidad del sistema educativo. Sin embargo, su visión de la modernización de la educación es profundamente humanista:

Cuando se anuncia la prioridad de la educación poniendo el acento de su fundamento en el hombre como agente del desarrollo económicosocial, se nos está diciendo algo que puede ser muy tremendo. Ciertamente que alcanzar determinada meta de ingreso per cápita en un cierto número de años pasa, inevitablemente, por un robustecimiento de la educación, por una mejor formación de sus sujetos, pero ella no se agota allí. Si esto no se ve claro, se nos empuja por una calle ciega, sin salida para las profundas aspiraciones humanas. La meta no es el bienestar en el tener, sino en la felicidad en el ser, en el vivir y en el con-vivir (LIVACIC, 1994, p. 237).

\section{Conclusiones}

Al cabo de haber examinado a Roberto Munizaga, representando el pensamiento laico, y a Ernesto Livacic, exponente del pensamiento confesional católico, se puede concluir que ellos mantienen una postura antagónica respecto de las creencias religiosas, pero que en cuanto a sus respectivos análisis sobre el papel que representa el proceso de educación en la vida del hombre muestran notables coincidencias. Y no podría ser de otra manera, por cuanto ambos autores, realizaron un trabajo profundo y 
desapasionado sobre el tema de la educación y sus propuestas están más apegadas a soluciones técnicas que a lemas ideológicos.

La mirada de los dos autores está de acuerdo en el rol indispensable que la escuela tiene en la formación del hombre y, a través de él, de la sociedad. La diferencia la pone el educador católico que prefiere hablar más bien de persona.

Ambos autores estiman que la manera de realizar el proceso educativo debe adecuarse a la situación del país, tomar en cuenta la idiosincrasia de los educandos y que debe considerárselo dentro de los factores productivos y de progreso social en su real importancia. Al momento de fundamentar este pensamiento, recurre cada cual a diversas fuentes, pero ambos coinciden en el filósofo norteamericano Dewey.

En lo meramente técnico, por ejemplo, organización curricular, papel de la educación preescolar y de la escuela, autonomía universitaria, podría decirse que hay una coincidencia casi total de ambos autores

La propuesta de ambos estudiosos conceden al educando su rol fundamental: el alumno es el responsable de su aprendizaje. Junto a él, el papel del profesor es insustituible. En este punto, a las características de mediador del conocimiento, de manejo de personas, de dominio de su oficio, Livacic agrega que el docente también tiene que ser un ejemplo moral para sus alumnos.

Finalmente, en ambos personajes están abordados a su manera los diferentes problemas filosófico-educacionales: el alumno como sujeto del proceso educativo; la familia, la escuela y el maestro como agentes externos al proceso educativo y que el desarrollo pleno del educando constituye el fin de la educación.

\section{Fuentes de consulta}

CAICEO, Jaime. Monseñor Oscar Larson Soudy (1892-1974). In: CELIS, Luis et al. Universidad Católica de Chile: Hombres e Ideas (1900-1950). Santiago: Pontificia Universidad Católica de Chile, 1988. p. 203-226.

. Influencia de Dewey en las Reformas Educacionales de Chile de 1927-1945. Santiago, Revista de Pedagogía, año XLIII, n. 356, p. 67-70, Maio. 1993. 
(1994) El Padre Hurtado y la Escuela Nueva en Chile.Revista de Educación, Santiago, n.220, p. 27-32, Set. 1994.

. Corrientes Filosóficas Presentes en la Historia de la Educación Chilena, Revista de Historia de la Educación, Santiago, v. 1, n. 1, p. 127132, 1995a.

(1995b) Influencia Pedagógica de Dewey en Chile. Asunción: Estudios Paraguayos, Assunção, v. XVIII, n. 1-2, p. 261-304, Dez. 1995b. . Algunos Antecedentes sobre la Presencia de la Escuela Nueva en Chile durante el Siglo XX. Buenos Aires: Anuario de Historia de la Educación, 2005.

HURTADO, Alberto (1994) El Sistema Pedagógico de John Dewey ante las Exigencias de la Doctrina Católica. 2. ed. Tradução de Jaime Caiceo. Santiago: Universidad Católica Blas Cañas.

CANALES, Manuel. Perfiles Biográficos del Profesor Ernesto Livacic Gazzano, Premio Nacional de Educación 1993, Foro Educacional, Santiago, v. 2, p. 130-145, 1997.

CELIS, Luis. Pensamiento Educacional de Roberto Munizaga: Un Intento de Innovación. In: SÁNCHEZ, Elena; CAICEO, Jaime et al. Filósofos y Educadores. El Pensar Chileno en el Siglo XX. Santiago: Universidad Católica de Chile, 1992.

KREBS, Ricardo et al. Catolicismo y laicismo. Las bases doctrinarias del conflicto entre la Iglesia y el Estado en Chile, 1875-1885: Seis estudios. Santiago: Ed. Universidad Católica de Chile, 1981.

LIVACIC, Ernesto. Panorama de la Educación Chilena a la Luz de la Participación. In: Pacífico, 1973. p. 45-70. . Educación y Participación en Chile. Santiago: . Los Idiomas Extranjeros y la Formación del Hombre. In:

Los Valores Formativos en las Asignaturas de la Enseñanza Media. Santiago: Nueva Universidad, 1979. p. 55-75.

. Amor y Educación. In: X Semana Social de Chile: La Eficacia del Amor. Santiago: Instituto Chileno de Estudios Humanísticos, 1982. p. 143-150. 
. Hacia una Acción Educativa Renovadora. In: SALMAN, Enrique et al. Hacia una Renovación Educativa Hoy. Santiago: San Pablo, 1993. p. 175-200. - Reformas Educacionales y Valores, Revista de Pedagogía, Santiago, año XLIV, n. 369, p. 235-241, Ago. 1994.

. La Educación, Signo de los Tiempos. Revista de la Universidad Metropolitana de Ciencias de la Educación, Santiago, v.1, n.1, p. 6-10, 1995.

. Algunos Aspectos de la Vocación de un Educador Cristiano, Revista de Pedagogía, Santiago, n. 384, p. 109-113, Marzo, 1996 .

. Palabra y Educación. In: . Foro Educacional. v. 1. Santiago: Universidad Católica Blas Cañas, 1996b. p. 15-25. 1997.

. Mario Hiriart. El Mensaje de un Laico. Santiago: Don Bosco, . Educación Escolar en Chile: Realidades, Desafíos, Horizontes. In: . Educación, Vocación y Compromiso. Santiago: Tiberíades, 2000.

MASSONE, Juan Antonio. Entrevista: Personajes de la Educación: El Enfoque Educativo de Ernesto Livacic G, Revista Educar, Santiago, ano 2, n. 20, p. 5-8, Abr. 1998.

MUNIZAGA, Roberto. Algunos Grandes Temas de la Filosofía de don Valentín Letelier. Santiago: Ed. Dirección General de Educación Primaria; Imprenta El Imparcial, 1943. . El Estado y la Educación. Santiago: Imprenta Universitaria, 1953. . (1958) En Torno a Sarmiento. Santiago: Ed. Universidad de Chile (es un Discuso de 1937 en la Escuela José A. Núñez; se publicó por primera vez en 1942 en la Revista de Educación).

- Libertad de Cátedra y Libertad de Investigación. Santiago: Departamento de Ciencias Sociales de la Facultad de Filosofía y Educación de la Universidad de Chile, 1964.

. Principios de Educación. 3. ed. Santiago: Universitaria, 1965.

. El Pensamiento Vivo de Letelier y los Actuales Problemas de una Reforma Universitaria (Separata Anales de la Universidad de Chile), año CXXVII, n. 150, Jurídica, Santiago Chile, 1969. 
Medio Siglo de Docencia Universitaria. La Serena: Ed. Universidad de Chile, 1978.

. Discurso Pronunciado en Vicuña el 11 de Julio de 1975 con Motivo de ser Declarado 'Hijo Ilustre' de la Ciudad. In: . Cinco Discursos sobre Educación. Santiago: Ed. Universitaria, 1984. La Filosofía Educacional de Froebel. In: . Dos Estudios sobre Educación. La Serena: Ed. Universidad de La Serena, 1986.

. Entrevista. Santiago: 16 de mayo. Entrevista concedida al autor del texto. 1990.

. Filosofía de la Educación Secundaria. 2. ed. Santiago: Ed. Universitaria, 1994. . et al. La Crisis Universitaria. Santiago: Imprenta Letras, 1933. ORTEGA Y GASSET, José. Misión de la Universidad. Madrid: Alianza Editorial, 1930

Data de registro: 05/12/2011 Data de aceite: $21 / 03 / 2012$ 
